\title{
How we approach thrombosis risk in children with COVID-19 infection
}

\author{
Anjali Sharathkumar ${ }^{1}$, E. Vincent Faustino ${ }^{2}$, and Clifford Takemoto ${ }^{3}$ \\ ${ }^{1}$ University of Iowa Children's Hospital \\ ${ }^{2}$ Yale School of Medicine \\ ${ }^{3}$ St. Jude Children's Research Hospital
}

January 25, 2021

\begin{abstract}
Thrombosis within the microvasculature and medium to large vessels is a serious and common complication among critically ill individuals with COVID-19. While children are markedly less likely to develop severe disease than adults, they remain at risk for thrombosis during acute infection and with the post-acute inflammatory illness termed multisystem inflammatory syndrome in children. Significant knowledge deficits in understanding COVID-19 associated coagulopathy and thrombotic risk pose clinical challenges for pediatric providers who must incorporate expert opinion and personal experience to manage individual patients. We discuss clinical scenarios to provide framework for characterizing thrombosis risk and thromboprophylaxis in children with COVID-19.
\end{abstract}

\section{Hosted file}

COVID_FINAL 1.1_CLEAN_01_20_2020.pdf available at https://authorea.com/users/391720/articles/ 505745-how-we-approach-thrombosis-risk-in-children-with-covid-19-infection

\section{Hosted file}

Table 1.pdf available at https://authorea.com/users/391720/articles/505745-how-we-approachthrombosis-risk-in-children-with-covid-19-infection

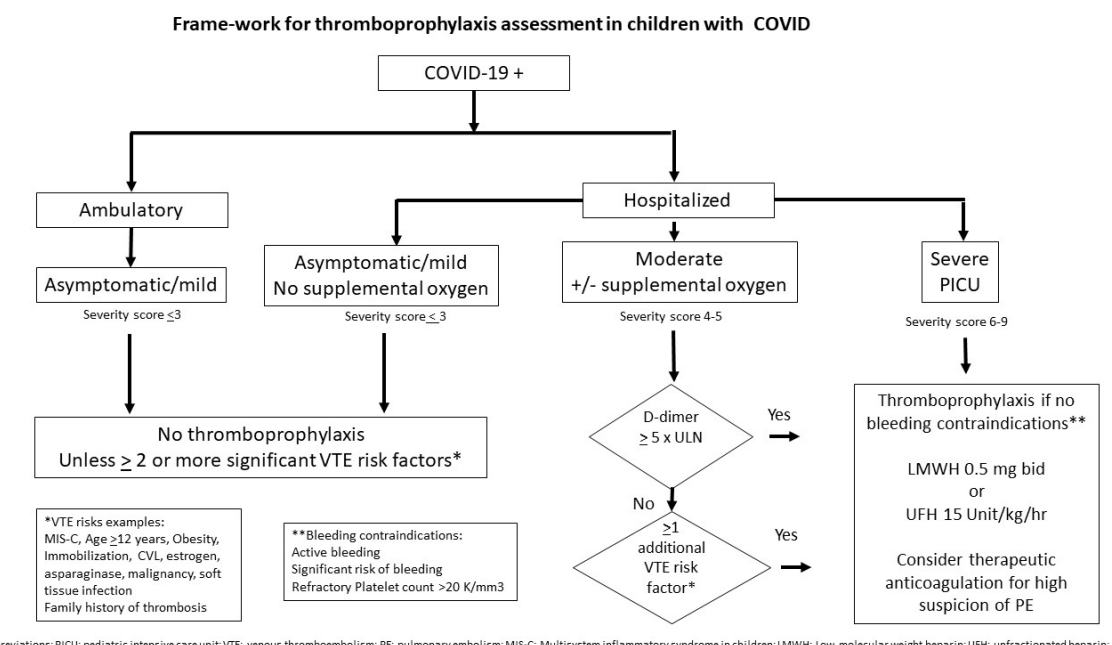

